\title{
Reliability of MRI for predicting characteristics of neurovascular conflicts in trigeminal neuralgia: implications for surgical decision making
}

\author{
Andrei Brînzeu, MD, MSc, PhD, ${ }^{1,2}$ Landry Drogba, MD, ${ }^{1,3}$ and Marc Sindou, MD, DSc ${ }^{1}$ \\ 1Department of Neurosurgery, University of Lyon 1, Lyon, France; ${ }^{2}$ Department of Neurosciences, Victor Babeş University of \\ Medicine and Pharmacy, Timişoara, Romania; and 'University of Medicine Abidjan, Yopougon, Abidjan, Côte d'Ivoire
}

\begin{abstract}
OBJECTIVE The choice of microvascular decompression (MVD), among the several other surgical options, for treating refractory classical trigeminal neuralgia (TN) relies mostly on preoperative imaging, but the degree of reliability of MRI remains a matter of debate. The authors approached the question of predictability of neurovascular conflict (NVC) in a series of 100 protocolized MRI studies from patients with TN who underwent MVD, by reexamination of MR images, blinded to the clinical data and surgical findings, including the side of the neuralgia.
\end{abstract}

METHODS Patients included in the study were those who underwent MVD after surgical indication had been determined based on a protocolized imagery workup (3D high-resolution T2-weighted cisternography centered on the trigeminal nerve, 3D time-of-flight angiography, and 3D gadolinium-enhanced T1-weighted imaging) performed at our institution. All MR images were blindly reexamined, and neurovascular relationships were described on both sides, noting the existence of compression, vessels involved, situation along the root, and degree of compression. The results of MRI evaluation were then compared with actual surgical findings. The extent of agreement and quality of the prediction were expressed with Cohen's kappa coefficient $(\kappa)$ and receiver operating characteristic $(\mathrm{ROC})$ statistics.

RESULTS A conflict had actually been found during surgery in 94 of 100 patients. The sensitivity of MRI to detect a conflict was $97 \%$ and the specificity was $50 \%$. Vessel type was identified with high reliability $(\kappa=0.80)$, while the grade of the conflict and its situation along the root showed poor to average reliability ( $\kappa=0.38$ and $\kappa=0.40$, respectively). The area under the ROC curve for predicting the presence of a conflict according to the grades of conflict seen on MRI was 0.93 , which is considered very good. The positive predictive value was differentiated according to the grade of conflict, with a very high value for high grades of vascular conflict.

CONCLUSIONS This study shows an overall good reliability of MRI to predict the existence of an NVC. The prediction value is excellent for high grades of compression. Some apparent low-grade compressions on MRI may be revealed as false positives in surgical exploration. This raises the question of what other imaging methods might be used to determine not only the existence of a conflict but also its degree of compression. The degree of compression is of paramount importance to predict the probability of long-term pain relief, and therefore in the decision to propose MVD as the first choice of surgical treatment.

https://thejns.org/doi/abs/10.3171/2017.8.JNS171222

KEYWORDS trigeminal neuralgia; neurovascular conflict; MRI evaluation; MRI predictability; microvascular decompression; decision making; functional neurosurgery; pain

I $\mathrm{N}$ determining whether to recommend microvascular decompression (MVD) to patients with trigeminal neuralgia (TN), it is important to be able to identify those who are likely to benefit from this treatment, since alternative treatment methods are available. This primarily relies on correctly predicting and evaluating potential neurovascular conflicts (NVCs), and therefore proper interpretation of MRI is the key element of the treatment decision. The aim of this study was to test the capability to predict and characterize neurovascular relationships (NVRs) in

ABBREVIATIONS AICA = anterior inferior cerebellar artery; BA = basilar artery; MVD = microvascular decompression; NVC = neurovascular conflict; NVR = neurovascular relationship; ROC = receiver operating characteristic; SCA = superior cerebellar artery; SPVS = superior petrosal venous system; TN = trigeminal neuralgia; TOF = time of flight; TREZ = trigeminal root entry zone; VA = vertebral artery.

SUBMITTED May 18, 2017. ACCEPTED August 1, 2017.

INCLUDE WHEN CITING Published online April 6, 2018; DOI: 10.3171/2017.8.JNS171222. 
patients with classical $\mathrm{TN}$ based on preoperative imaging studies, with the examiner being blinded to clinical and surgical data, including the side of the neuralgia.

A considerable amount of experience has been accumulated on the treatment of TN by MVD, with many published series showing favorable long-term results. However, the method is far from being a panacea, and studies focusing on long-term outcome report a significant failure or recurrence rate even after well-conducted MVD, to the point that even the fundamentals of the method, i.e., the key role of NVCs in the pathogenesis of $\mathrm{TN}$, have been ${ }^{1}$ and are still contested. ${ }^{7}$

In a previous study on patients selected for MVD based on MRI (i.e., showing NVRs likely to correspond to an NVC), failure rates were foremost associated with a low grade of conflict (i.e., contact without clear-cut alteration of the root at surgery). ${ }^{38}$ Kaplan-Meier analysis in that study showed that the probability of freedom from pain at 15 years postoperatively in patients who had grade I compression (simple contact) was $65 \%$, whereas the probability was $73 \%$ for patients with grade II (displacement of the nerve) and $85 \%$ for those with grade III (indentation of the nerve).

Because of the availability of viable percutaneous or radiosurgical alternatives for the treatment of refractory $\mathrm{TN}$, it is important to be able to exclude from MVD indication patients in whom a low probability of long-term pain relief could be predicted on MRI examination.

Many publications have stressed the high sensitivity and specificity of high-resolution MRI to detect NVC.,23, ${ }^{29,30}$ The degree of the vascular compression can also be estimated on these imaging studies, but to a lesser extent. On the other hand, MRI may show close relationships between neighboring vessels and the trigeminal root in individuals without $\mathrm{TN}$ or on the asymptomatic side of patients with TN. ${ }^{7,31}$ Occasionally in some patients presenting with classical TN and with a suggestive MRI, no NVC is found at surgical exploration. ${ }^{20}$ This raises the question of the predictive value of MRI in a typical clinical setting.

The present study was designed to not only further explore the predictive value of MRI regarding the presence of NVC but also to identify the responsible vessel(s) and determine the degree of compression. Imaging studies from 100 consecutive patients with classical TN who had benefited from MVD, performed based on an MRI considered to be positive for an NVC, were retrospectively reviewed by an experienced examiner blinded to the clinical and surgical findings, including the side of the neuralgia. Comparison of estimations with the operative findings is the subject of this article.

\section{Methods}

\section{Patient Selection}

Data were collected from patients with classical TN in whom, based on preoperative imaging, a probable NVC was suspected and MVD was carried out at the Pierre Wertheimer Hospital in Lyon, France, in the period from 2000 to 2014. Patients in whom an image of NVC was not seen on preoperative MRI were not operated on by MVD (an alternative technique was proposed to them per institu- tion policy) and therefore not included in this study. Only the patients who underwent a complete preoperative MRI workup at our institution were included. All patients who had undergone a previous trigeminal nerve surgery were excluded. Patients who underwent not only MVD of the trigeminal nerve but also MVD of 1 or more other cranial nerves were also excluded, as were patients with bilateral neuralgia.

All patients were referred to our institution for surgery after failure of medical treatment or development of major side effects, including severe fatigue (described as insufficient energy to perform daily activities). ${ }^{6,39} \mathrm{In}$ each case, the diagnosis of classic TN was confirmed according to the ICHD (International Classification of Headache Disorders) ${ }^{25}$ criteria and was compliant with newer classic TN diagnostic and classification criteria. ${ }^{11,28}$

All the patients included in the study underwent MRI examinations using the standardized protocol previously published. ${ }^{22,23}$ Per protocol, all of these examinations included 3 high-resolution sequences centered on the trigeminal root: 3D T2-weighted driven equilibrium, 3D time-of-flight (TOF) angiography, and 3D T1-weighted sequences with gadolinium.

The indication for surgery was based on the identification of a potential NVC of at least grade I according to our institution's anatomical classification (shown in Table 1). ${ }^{37}$ MR images were reviewed after the patients were found to meet the inclusion criteria. In all cases, the review was conducted at least 2 years after the surgical procedure.

\section{Surgical Technique}

MVD was performed in all cases by the senior author (M.S.) according to a previously published, standardized microsurgical technique. ${ }^{37,38}$ No attempt at intentionally lesioning the nerve was performed, even in patients in whom no clear-cut conflict was found at surgery. The key point of the surgery was complete exploration of the trigeminal root from the brainstem to the porus trigeminus of Meckel's cave, through a retromastoid-retrosigmoid approach and an infratentorial-supracerebellar intradural approach. Operative reports included the compressive vessel(s), the situation of the conflict(s) along the root (i.e., the relationship to the root in terms of anatomical direction), and-importantly-the degree of compression.

\section{Study Design}

MR images and operative reports were collected for the included cases. A database was then constructed by separate investigators, with one component for the surgical findings (A.B.) and the other for the MR study (L.D.). The MR images were evaluated by an experienced surgeon (the senior author, M.S.), while he was blinded to surgical and clinical data, including the side of the neuralgia.

\section{Blinded MRI Examination}

NVRs of the bilateral trigeminal nerves were evaluated in each case. Any vessel that was deemed to present potential for NVC along the trigeminal root was noted, as was the precise site of potential conflict. Each conflict was then graded according to a previously described scale 
TABLE 1. Surgical and imaging descriptions of the different grades of compression

\begin{tabular}{cll}
\hline Grade & \multicolumn{1}{c}{ Surgical Description* } & \multicolumn{1}{c}{ Imaging Description† } \\
\hline 0 & Neurovascular relation without contact & Neurovascular relation without contact \\
\hline I & Simple contact without visible alteration of the root & Contact: absence of interposed CSF layer \\
\hline II & Displacement/distortion of the root & Deviation of the root \\
\hline III & Indentation of the root/engrooving/focal demyelination & Indentation of the root \\
\hline
\end{tabular}

In this grading system, veins are graded according to the same scale as arteries.

* Based on Sindou et al., 2007.

† Based on Leal et al., 2010.

(see Table 1). ${ }^{23}$ Once the conflict was graded for each involved vessel, patients were classified as showing unilateral or bilateral NVRs. Bilateral NVRs were divided into symmetrical and asymmetrical, with symmetrical NVR defined as the presence of the same type of vessel with an approximately similar zone of contact and relatively the same anatomical impact or deformation of the root.

Then the groups of bilateral symmetrical and asymmetrical conflicts were compared and statistically analyzed by calculating the concordance coefficient (Cohen's kappa statistic) for vessel type on one side versus the other and the grade of the conflict.

Finally, an attempt was made to identify (i.e., predict) the side of the neuralgia while still blinded to all of the patient's data.

\section{Comparison With Surgical Findings}

Next, the images of the conflicting vessel(s), as described on blinded MRI examination, were compared with those found at surgery. The grading of the conflicts was compared, noting the differences (over- or undergrading). The site of the actual conflict was compared with the one described on MRI examination. Finally, the blinded estimation of the side considered neuralgic was compared with the actual side of the TN.

\section{Data Analysis}

The predictive value of MRI was first evaluated by calculating the sensitivity, specificity, positive predictive value, and accuracy for the prediction of the existence of an NVC on the side of the neuralgia after comparison with the surgical findings.

Cohen's kappa ${ }^{10,18}$ was used to assess the predictive value of MRI for each specific vessel by calculating the concordance between estimation of the vessel seen on MRI and the surgical findings. As previously described, ${ }^{23}$ Cohen's kappa reduces the impact of chance when assessing the quality of a diagnostic test for categorical values. Values $\leq 0.2$ are considered poor; values $>0.2$ and $\leq 0.4$, average; values $>0.4$ and $\leq 0.6$, good; and values $>0.6$ and $\leq 0.8$, excellent. Values $>0.8$ indicate results that approach perfect diagnostic prediction. The same measure was used to evaluate the quality of prediction for the situation of the conflict and the degree of compression.

To assess the overall predictive value of MRI for the existence of a conflict, a receiver operating characteristic (ROC) space was defined using the grading of each of the NVRs as seen on MRI and comparing it with the presence or absence of an actual conflict (in binary form-that is conflict present or not) as found at surgery.

ROC curves are a tool used to evaluate the quality of a diagnostic test. ${ }^{21,40,41}$ The curves are constructed by plotting (in a $1 / 12 \mathrm{D}$ space) the rate of true positives (sensitivity) against the rate of false positives $(1-$ specificity). This provides a composite measure of the performance of a diagnostic test as a binary classifier (here, distinguishing between patients with and without true conflicts). The area under the ROC curve quantifies the quality of the test, with values varying from 0.5 (when the diagnostic value of the test would be equal to that of random chance-i.e., no diagnostic value, illustrated by the diagonal of the ROC space) to 1 (a perfect fit, illustrated by the upper horizontal line, when the classifier commits no errors). Values above 0.85 are considered excellent.

Curves were calculated using all the conflicts predicted on MRI and using intraoperative observation as the gold standard to define the existence of an actual NVC. Specifically, for each patient, the sum of the grades of all conflicts seen on MRI was used to calculate data points for ROC curves. Different cutoffs were used for the surgical diagnosis of conflict according to the grade found at surgery (i.e., whether grade I, II, or III as found at surgery was considered to be a true conflict). The different curves were compared for statistical difference using methods previously described. ${ }^{19}$

Grades of conflict were converted to Arabic numerals for calculating mean values.

\section{Side of the Neuralgia}

Prediction of the side of the neuralgia was analyzed at the level of the entire study population as well as between the groups and subgroups of patients with unilateral or bilateral (symmetrical or asymmetrical) conflicts. The error rate in predicting the side of the neuralgia was compared between the 3 categories.

\section{Results \\ Demographics}

Of the 100 patients included in the study, 54 were men and 46 were women. Their mean $( \pm$ SD) age at the time of surgery was $52 \pm 12$ years. These patients had been suffering from TN for an average of $4.5 \pm 1$ years prior to surgery. The distribution of neuralgia is given in Table 2. In 49 patients, the pain was purely paroxysmal, while in the rest of them the paroxysmal component either was associ- 
TABLE 2. Distribution of TN by trigeminal nerve territory affected

\begin{tabular}{cc}
\hline Territory & Distribution (\%) \\
\hline V1 & 7.14 \\
\hline V2 & 20.23 \\
\hline V3 & 16.66 \\
\hline V1, V2 & 13.09 \\
\hline V2, V3 & 33.33 \\
\hline V1, V2, V3 & 9.52 \\
\hline V1 all & 30 \\
\hline V2 all & 76 \\
\hline V3 all & 59 \\
\hline
\end{tabular}

ated with a continuous background pain $(n=33)$ or had been completely replaced by the continuous background pain following the evolution of the pain and/or the introduction of medical treatment, although it had been present at the beginning of the disease $(n=18)$.

\section{Operative Findings}

At surgery, 94 of the 100 patients had a clear-cut NVC between the trigeminal nerve and at least one of the neighboring vessels. In 6 patients, no conflict was found. In 33 patients, multiple conflicts were discovered. The arterial conflicting vessels were as follows: superior cerebellar artery (SCA) in 75 cases, anterior inferior cerebellar artery (AICA) in 12 cases, and vertebral artery or basilar artery (VA/BA, considered a continuum in this study) in 4 cases. Veins were identified as conflicting vessels in 36 cases: as the sole conflicting vessel in 11 cases and exerting a strong compression in association with an artery in 25 cases. Association of a vein and an artery was found to be more frequent than arteries associated in multiple conflicts; there were 8 multiple conflicts involving 2 arteries $(\mathrm{p}<0.000012$, Fisher's exact test).

In total, 127 vessels were found to be involved in NVCs at surgery. The conflicts were classified as grade I for 24 vessels, grade II for 47, and grade III for 56. Details of surgical findings are given in Table 3.

Arterial conflicts were found to be of a significantly higher grade than venous ones ( $p=0.00025$, Fisher's exact test). No significant difference was found between the grading of the deep and superficial venous conflicts $(\mathrm{p}=$ 0.526 , Fisher's exact test).

The SCA, which was found to be involved in NVC 75 times at surgery, was the sole conflicting vessel in 42 patients (i.e., $56 \%$ of all cases of SCA conflicts found). SCA conflicts were mostly grade II (35 patients; $47 \%$ ) or grade III (34 patients; 45\%), with the conflict occurring most often in the cisternal portion (67\%). The AICA was found to be involved in 12 patients-and as the sole conflicting vessel in $6(50 \%)$. Conflicts with the AICA occurred for the most part (67\%) at the trigeminal root entry zone (TREZ) and never at the porus, with a predominance of higher grades ( $75 \%$ grade II or III). These findings are similar to those for the VA/BA. The location of the conflict was found to differ significantly according to vessel type. Conflict with the SCA occurred mostly in the cisternal portion, while conflict with the AICA occurred mostly at the TREZ ( $p=0.00006$, Fisher's exact test).

The superficial superior petrosal venous system

TABLE 3. Surgical findings and assessments based on MRI evaluation

\begin{tabular}{|c|c|c|c|c|c|c|c|c|}
\hline \multirow[b]{2}{*}{ Method \& Vessel } & \multirow[b]{2}{*}{ Total } & \multicolumn{3}{|c|}{ Grade of Compression } & \multicolumn{3}{|c|}{ Site of NVC } & \multirow[b]{2}{*}{ Unique* } \\
\hline & & I & II & III & TREZ & Cisternal & Porus & \\
\hline \multicolumn{9}{|l|}{ Surgical exploration } \\
\hline SCA & 75 & $6(8)$ & $35(47)$ & $34(45)$ & $21(28)$ & $50(67)$ & $4(5)$ & $42(57)$ \\
\hline AICA & 12 & $3(25)$ & $3(25)$ & $6(50)$ & $8(67)$ & $4(33)$ & 0 & $6(50)$ \\
\hline VA/BA & 4 & $1(25)$ & 0 & $3(75)$ & $3(75)$ & $1(25)$ & 0 & $2(50)$ \\
\hline sSPVS & 18 & $9(50)$ & $4(22)$ & $5(28)$ & $9(50)$ & $9(50)$ & 0 & $5(28)$ \\
\hline dSPVS & 18 & $5(28)$ & $6(33)$ & $7(39)$ & 0 & $3(17)$ & $15(83)$ & $6(33)$ \\
\hline \multicolumn{9}{|l|}{ Ipsilateral MRI evaluation } \\
\hline SCA & 80 & $27(33.7)$ & $34(42.5)$ & $19(23.75)$ & $18(22.5)$ & $60(75)$ & $1(1.25)$ & $46(57.5)$ \\
\hline AICA & 6 & $2(33.3)$ & $2(33.3)$ & $2(33.3)$ & $5(83.3)$ & $1(16.7)$ & 0 & $3(50)$ \\
\hline VA/BA & 4 & $0(0)$ & $0(0)$ & $4(100)$ & $3(75)$ & $1(1)$ & $0(0)$ & $1(25)$ \\
\hline sSPVS & 28 & $25(89.2)$ & $3(10)$ & $0(0)$ & $10(35.7)$ & $19(67.8)$ & $0(0)$ & $6(21.4)$ \\
\hline dSPVS & 9 & $4(44.4)$ & $4(44.4)$ & $1(11.1)$ & $0(0)$ & $3(33.3)$ & $6(66.7)$ & $3(33.3)$ \\
\hline \multicolumn{9}{|c|}{ Contralateral MRI evaluation } \\
\hline SCA & 35 & $22(62.8)$ & $13(37.3)$ & $0(0)$ & $6(17.1)$ & $29(82.8)$ & 0 & $30(85.7)$ \\
\hline sSPVS & 7 & $6(85.7)$ & $1(14.2)$ & $0(0)$ & $5(71.4)$ & $2(28.5)$ & 0 & $2(28.5)$ \\
\hline dSPVS & 7 & $4(57.1)$ & $3(42.8)$ & $0(0)$ & $0(0)$ & $2(28.5)$ & $5(71.4)$ & $7(100)$ \\
\hline
\end{tabular}

dSPVS = deep SPVS; sSPVS = superficial SPVS.

Data are presented as number (\%) and indicate cases in which the specified vessel either was found to be involved at surgical exploration or was thought to be involved based on MRI evaluation.

* Refers to a single (vs multiple) conflict. 


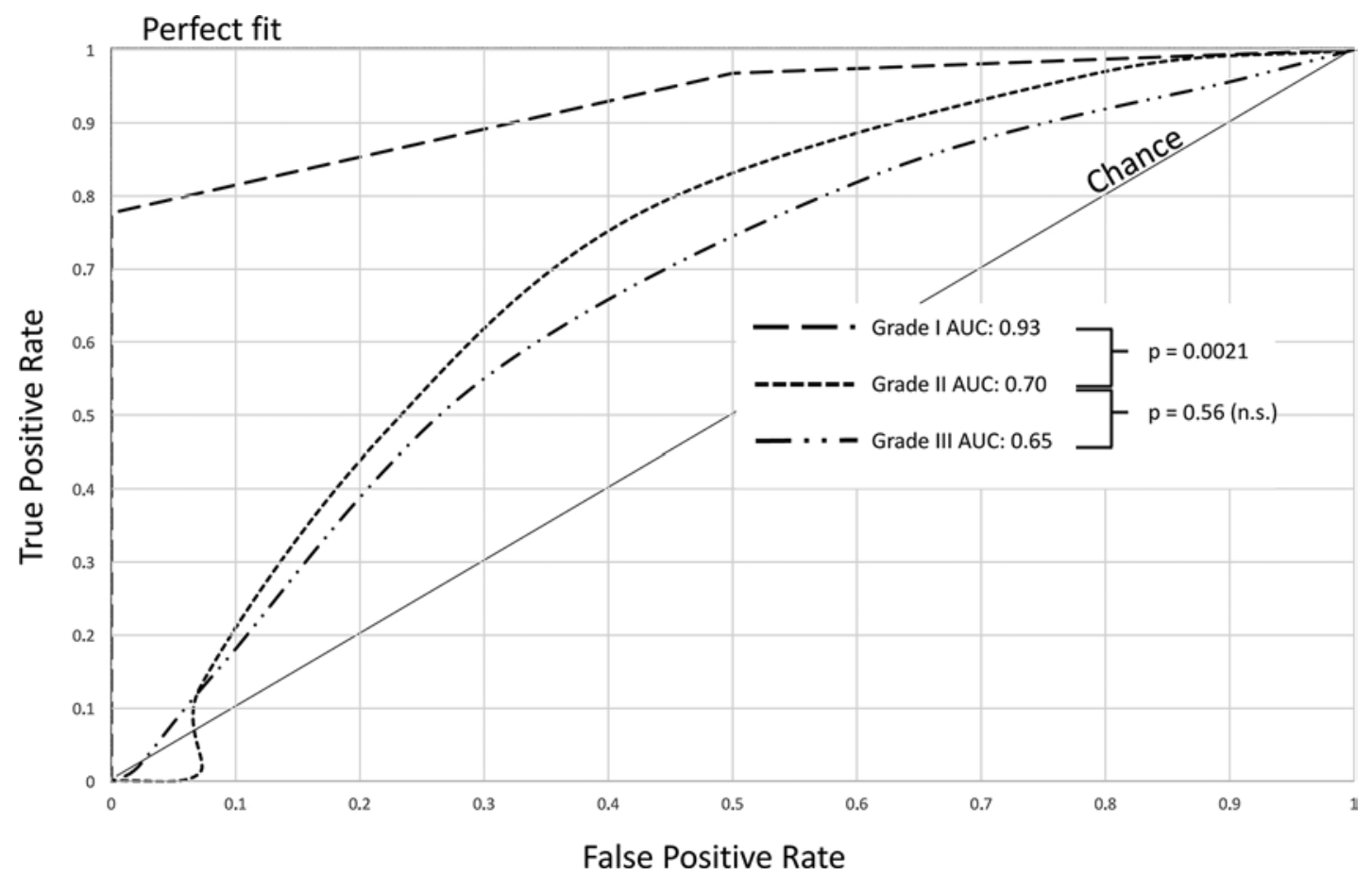

FIG. 1. ROC curves for different definitions of NVC. n.s. = not significant.

(SPVS) ${ }^{16}$ was found to be involved in 18 patients, for the most part (in 13 cases [72\%]) as a second conflicting vessel associated with an artery. Conflicts involving the superficial SPVS occurred either at the TREZ $(50 \%)$ or in the cisternal portion (50\%) but never at the porus. Half of the conflicts were estimated to be of grade I; this is the highest proportion of grade I conflicts among all the conflicting vessels found at surgery. Veins of the deep SPVS ${ }^{13}$ were also found to be involved 18 times, also predominantly in association with other arterial conflicts (in 12 cases [66\%]) and with an even distribution among grades. Their location was predominantly at the porus of Meckel's cave $(\mathrm{n}=15,83 \%)$, and the difference in frequency (in comparison with other locations) was statistically significant $(\mathrm{p}=0.00007$, Fisher's exact test).

\section{Description of NVRs by Blinded MRI Examination}

At blinded MRI examination, including both sides, 176 NVRs were described. Data on the specific vessels involved, the location along the root, and the estimated grade of compression are presented in Table 3 .

Blinded examination identified unilateral NVRs in 56 patients and bilateral NVRs in 44. Based on analysis of the MRI data, patients with bilateral potential conflicts were divided into 2 groups: one with symmetrical conflicts, i.e., apparently similar vessel types and grades on both sides (n $=30$ ), and the other with asymmetrical conflicts, i.e., with a predominant conflict on 1 side $(n=14)$. A comparison of the grade of conflict between the neuralgic and the nonneuralgic sides is given in Fig. 1.

The 2 groups with bilateral NVRs were different in terms of vessel type on one side and the other, with a statistically significant difference in their Cohen's kappa values-0.21 (95\% CI 0.18-0.24) in the asymmetrical group and 0.87 (95\% CI 0.86-0.88) in the symmetrical group (p $=0.001$ ). There was also a significantly higher grade of conflict on the side of the neuralgia for the asymmetrical group (mean 1.68 vs $0.72, p=0.001$, Wilcoxon rank-sum test). Comparisons of different subgroups of the patients with bilateral conflicts are presented in Table 4 .

\section{Sensitivity, Specificity, and Positive Predictive Value of Blinded MRI Examination}

Through blinded examination of the MR images, an NVR was identified in 94 patients on the side of the neuralgia. In the other 6 patients, it did not result in the identification of a possible conflict on the side of the neuralgia. Of these 6 patients, 3 had no conflict at surgery, whereas the other 3 did have a small-grade conflict (i.e., false negative). In 3 of the 94 patients in whom a conflict was identified on MRI, no conflict was found at surgery (i.e., false positives). As previously mentioned, an NVC was found at surgery in only 94 patients.

Based on these data, the overall sensitivity of blinded MRI examination was calculated to be $97 \%$; the specificity, $50 \%$ (half of the patients without a conflict were thought to have one); the positive predictive value, $97 \%$ (a conflict identified on MRI was confirmed at surgery); and the overall accuracy, 97\% (Table 5).

Sensitivity, specificity, positive predictive value, and accuracy are given in Table 5 for the global capacity of a conflict to be identified through blinded MRI examination, as well as for the identification of each specific vessel.

The value of Cohen's kappa for overall vessel identification was 0.68 (95\% CI 0.65-0.71). Values for each vessel are given in Table 5, with the values being good or very good for the VA/BA, SCA, and veins, but not as good for the AICA $(\kappa=0.40,95 \%$ CI 0.38-0.42). 
TABLE 4. Comparisons of results of blinded MRI evaluations in the patients with bilateral conflicts

\begin{tabular}{|c|c|}
\hline Variable & Value \\
\hline Asymmetrical findings (no. of patients) & 14 \\
\hline Ipsilateral MRI (no. of conflicts) & 22 \\
\hline \multicolumn{2}{|l|}{ Grade of conflict } \\
\hline Mean* & 1.68 \\
\hline Grade I & $50.00 \%$ \\
\hline Grade II & $31.82 \%$ \\
\hline Grade III & $18.18 \%$ \\
\hline \multicolumn{2}{|l|}{ Site } \\
\hline TREZ & $36.36 \%$ \\
\hline Cisternal & $54.54 \%$ \\
\hline Porus & $9.09 \%$ \\
\hline Multiple & $9 \%$ \\
\hline \multicolumn{2}{|l|}{ Vascular conflict } \\
\hline SCA & $54.55 \%$ \\
\hline AICA & $9.09 \%$ \\
\hline VA/BA & $4.55 \%$ \\
\hline SSPVS & $22.73 \%$ \\
\hline dSPVS & $9.09 \%$ \\
\hline Contralateral MRI (no. of conflicts) & 15 \\
\hline \multicolumn{2}{|l|}{ Grade of conflict } \\
\hline Mean* & 0.72 \\
\hline Grade I & $93.33 \%$ \\
\hline Grade II & $6.67 \%$ \\
\hline Grade III & $0.00 \%$ \\
\hline \multicolumn{2}{|l|}{ Site } \\
\hline TREZ & $13.33 \%$ \\
\hline Cisternal & $66.67 \%$ \\
\hline Porus & $20 \%$ \\
\hline Multiple & $1 \%$ \\
\hline \multicolumn{2}{|l|}{ Vascular conflict } \\
\hline SCA & $66.67 \%$ \\
\hline AICA & $0.00 \%$ \\
\hline VA/BA & $0.00 \%$ \\
\hline sSPVS & $13.33 \%$ \\
\hline dSPVS & $20.00 \%$ \\
\hline Symmetrical (no. of patients) & 30 \\
\hline Ipsilateral MRI (no. of conflicts) & 34 \\
\hline \multicolumn{2}{|l|}{ Grade of conflict } \\
\hline Mean* $^{*}$ & 1.71 \\
\hline Grade I & $64.71 \%$ \\
\hline Grade II & $29.41 \%$ \\
\hline Grade III & $5.88 \%$ \\
\hline \multicolumn{2}{|l|}{ Site } \\
\hline TREZ & $8.82 \%$ \\
\hline Cisternal & $88.24 \%$ \\
\hline Porus & $2.94 \%$ \\
\hline Multiple & $4 \%$ \\
\hline
\end{tabular}

CONTINUED IN NEXT COLUMN »
» CONTINUED FROM PREVIOUS COLUMN

TABLE 4. Comparisons of results of blinded MRI evaluations in the patients with bilateral conflicts

\begin{tabular}{|c|c|}
\hline Variable & Value \\
\hline Symmetrical (no. of patients) (continued) & 30 \\
\hline Ipsilateral MRI (no. of conflicts) (continued) & 34 \\
\hline \multicolumn{2}{|l|}{ Vascular conflict } \\
\hline SCA & $73.53 \%$ \\
\hline AICA & $0.00 \%$ \\
\hline VA/BA & $0.00 \%$ \\
\hline SSPVS & $17.65 \%$ \\
\hline dSPVS & $8.82 \%$ \\
\hline Contralateral MRI (no. of conflicts) & 34 \\
\hline \multicolumn{2}{|l|}{ Grade of conflict } \\
\hline Mean* & 1.47 \\
\hline Grade I & $52.94 \%$ \\
\hline Grade II & $47.06 \%$ \\
\hline Grade III & $0.00 \%$ \\
\hline \multicolumn{2}{|l|}{ Site } \\
\hline TREZ & $26.47 \%$ \\
\hline Cisternal & $67.65 \%$ \\
\hline Porus & $5.88 \%$ \\
\hline Multiple & $40 \%$ \\
\hline \multicolumn{2}{|l|}{ Vascular conflict } \\
\hline SCA & $73.53 \%$ \\
\hline AICA & $0.00 \%$ \\
\hline VA/BA & $0.00 \%$ \\
\hline sSPVS & $14.71 \%$ \\
\hline dSPVS & $11.76 \%$ \\
\hline
\end{tabular}

\section{Situation of the NVRs Along the Root}

The overall accuracy of the estimation of the sites of conflict along the trigeminal root was calculated to be $53.5 \%$. This was best for conflicts located in the cisternal portion of the nerve $(70 \%)$, while it was $42.5 \%$ for conflicts located at the TREZ and only $20 \%$ for conflicts located at the porus of Meckel's cave. Exact figures with calculated positive predictive value for each of the locations are given in Table 5. The value of Cohen's kappa for the site of conflict along the root was 0.37 (95\% CI $0.33-0.41)$ due to lack of strict anatomical differentiation between the TREZ and the cisternal portion of the nerve $(\kappa=0.20$ for the difference between the two).

\section{Grades of Conflict}

The mean value of the grade of conflict estimated after MRI examination was 1.54 , whereas at surgery this was found to be 1.97. The difference of 0.42 was found to be significant (Wilcoxon rank-sum test, $\mathrm{p}=0.00011$ ), testifying to an overall underestimation of the grade of conflict by MRI.

The degree of conflict found at surgery was underestimated in $45 \%$ of all conflicts, exactly estimated in $33 \%$, 
TABLE 5. Vessel detection sensitivity and specificity

\begin{tabular}{lcccccc}
\hline Variable & Sensitivity & Specificity & PPV & NPV & Accuracy & $\kappa$ \\
\hline Global & $0.97(0.91-1.00)$ & $0.5(0.12-0.88)$ & $0.97(0.94-0.99)$ & $0.5(0.2-0.8)$ & $0.94(0.87-0.98)$ & $0.68(0.65-0.71)$ \\
\hline SCA & $0.88(0.79-0.95)$ & $0.72(0.50-0.89)$ & $0.92(0.85-0.96)$ & $0.64(0.48-0.78)$ & $0.78(0.67-0.86)$ & $0.78(0.75-0.81)$ \\
\hline AICA & $0.67(0.22-0.96)$ & $0.91(0.84-0.96)$ & $0.33(0.17-0.54)$ & $0.98(0.94-0.99)$ & $0.94(0.87-0.98)$ & $0.40(0.38-0.42)$ \\
\hline VA/BA & 1 & 1 & 1 & 1 & 1 & 1 \\
\hline Veins & $0.85(0.73-0.93)$ & $0.7(0.46-0.81)$ & $0.74(0.59-0.84)$ & $0.77(0.69-0.84)$ & $0.72(0.60-0.85)$ & $0.81(0.79-0.83)$ \\
\hline
\end{tabular}

NPV = negative predictive value; $\mathrm{PPV}=$ positive predictive value.

Values in parentheses are $95 \%$ confidence intervals.

and overestimated in $22 \%$. Eighty percent of overestimations occurred in patients who were found at surgery to have either a low-grade (grade I) conflict or no conflict, while $90 \%$ of underestimations were in cases that turned out to be grade II or III conflicts.

The positive predictive value for the presence of a conflict was evaluated for each of the grades. For conflicts classified as grade I, it was 77\%; for grade II, 90\%; and for grade II, $100 \%$. When a high grade (II or III) was seen on the MRI, its positive predictive value was $95 \%$.

The sensitivity for detecting the presence of a grade I conflict was lowest, at $70 \%$, while the sensitivity was $89 \%$ for grade II and $90 \%$ for grade III. The specificity for detecting the absence of a conflict overall (grades I-III) was $50 \%$. When only conflicts graded II or III were considered, the specificity was $100 \%$. Cohen's kappa for overall concordance of degree of conflict predicted on MRI and found at surgery was 0.356 (95\% CI $0.350-0.362)$.

ROC curves calculated for cutoffs at grades I, II, and III are given in Fig. 2. The area under the ROC curve for the grade I cutoff was found to be $0.93 \pm 0.0331$, the area for the grade II cutoff was $0.70 \pm 0.0673$, and the area for the grade III cutoff was $0.65 \pm 0.056$. The area under the curve for the grade I cutoff was significantly higher than that for grade II or III $(p=0.0021)$, whereas there was no statistically significant difference between the area under the curve values for the grade II and III cutoffs. Figure 2 shows the different ROC curves.

\section{Prediction of the Side of Neuralgia}

In 19 cases the side of the neuralgia was not correctly predicted at blinded MRI examination. Prediction was erroneous in 13 of the patients whose MR images were interpreted as showing bilateral symmetrical conflicts, 3 of the 14 patients whose images were interpreted as showing bilateral asymmetrical conflicts, and 3 of the 56 patients whose images were interpreted as showing unilateral conflicts. The rate of error was significantly higher for the patients with bilateral conflicts identified on MRI

$$
p=0.00007
$$
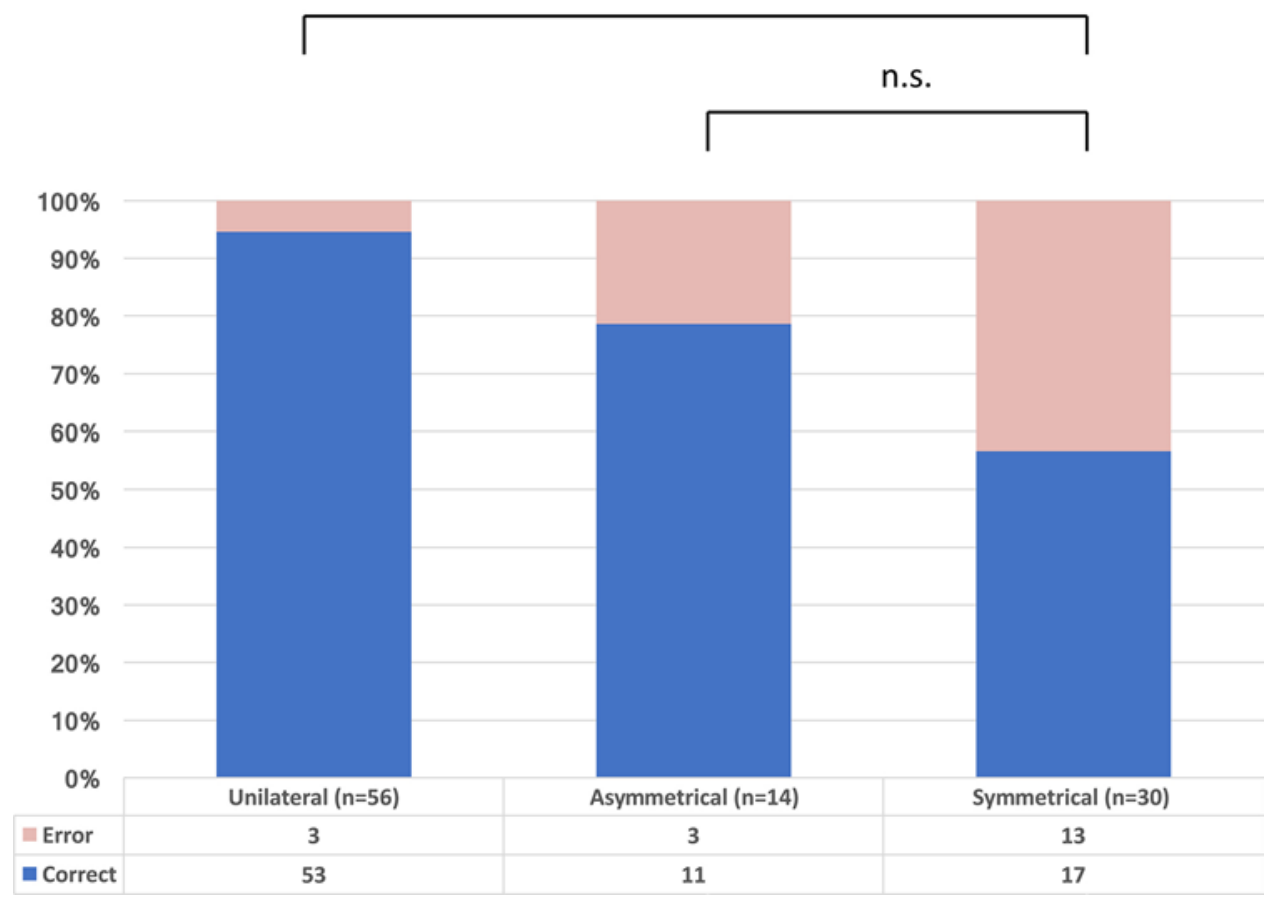

FIG. 2. Errors in predicting the side of neuralgia. Figure is available in color online only. 


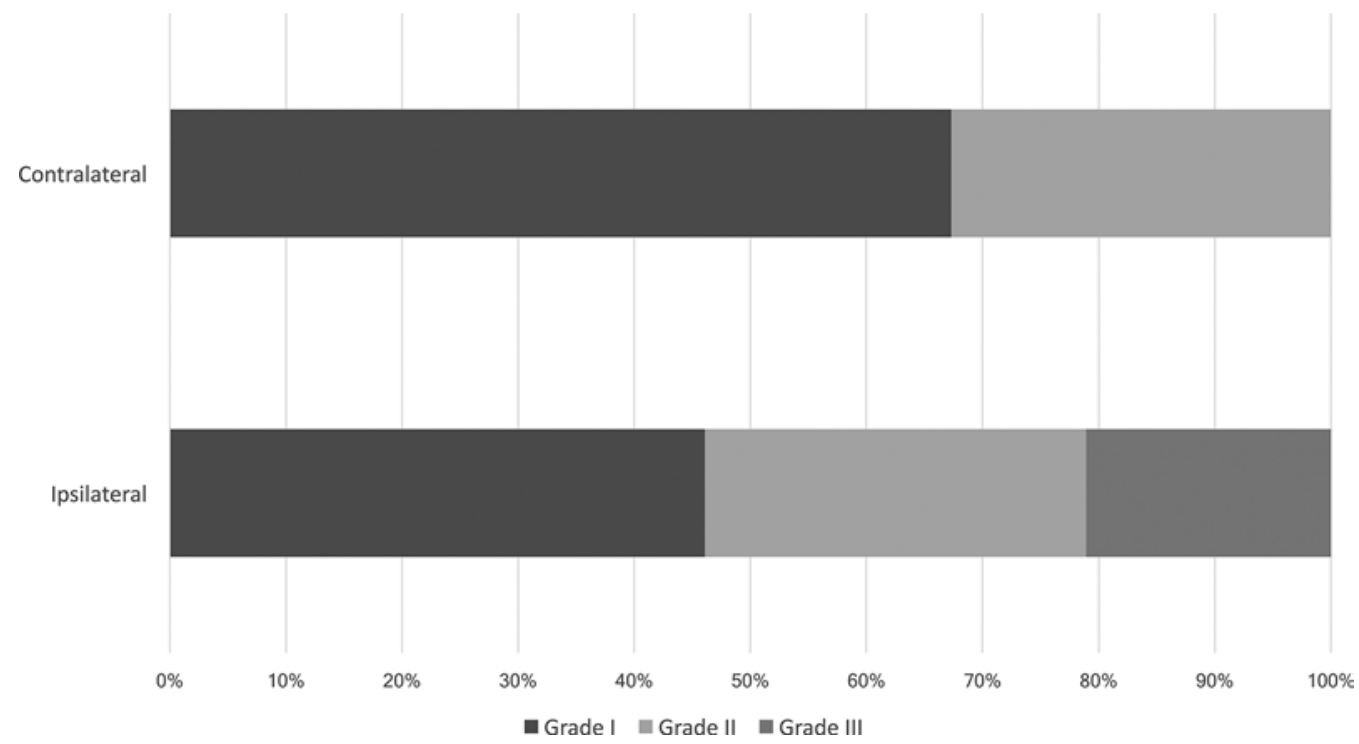

FIG. 3. Comparison of the grading of conflicts on the symptomatic and asymptomatic sides in patients with bilateral conflicts.

( $p=0.00008$, chi-square test), but no difference was found between the symmetrical and asymmetrical groups ( $\mathrm{p}=$ 0.19 , Fisher's exact test). Figure 3 shows the differences in errors in predicting the side of the neuralgia.

\section{Discussion}

This study is based on blinded reexamination of MRI studies of a large series of patients who had already been determined to have a surgical indication for MVD on that very MRI. One hundred institutionally protocolized MRI studies $^{23}$ were collected, with all of these studies having been performed in patients with classic TN in whom a conflict was thought to be very likely at the time that the indication for surgery was determined. These MRI studies were reexamined years after the surgery by an examiner who was blinded to the clinical and surgical findings and unaware of the side of the neuralgia. The examiner (who was also the surgeon who performed all of the decompressions) knew, however, that all MR images were considered positive before the surgery, thus prompting him to find a conflict at least on one side. Under these conditions, the sensitivity of MRI to detect conflicts was $97 \%$. More importantly, the specificity when reexamining imagery was $50 \%$. This means that $50 \%$ of the patients in whom a surgical indication had been identified, based on an MRI considered positive initially but without an actual conflict at surgery, were identified as negative (true negatives) on MRI reexamination. Overall, the quality of prediction for the existence of a conflict, defined as at least grade I (simple contact), based on MRI, was excellent, with a low rate of false positives, leading to an area under the ROC curve of 0.93 .

The choice of collecting data from a single examiner was purposely made; despite the potential bias, this choice best replicates the clinical situation when the decision for surgery is usually made by a single responsible surgeon. The combination of the use of only a single examiner and the long experience of that examiner led to a homoge- neous set of data collected with valuable precision. This was tested by randomly selecting 10 patients who were again blindly reexamined at the end of the study. The test revealed a perfect concordance with the initial blinded estimation ( $\kappa=1$ for comparison of all elements: vessel type, conflict location, and conflict grade; data not shown), leading to a coherent data set.

Vessel identification was for the most part of very good quality with concordance coefficients (Cohen's kappa values) of 0.8 .

The exact nature (arterial or venous) of the conflicting vessels and their exact origin (i.e., SCA, AICA, or VA/BA for arteries and the superior or deep SPVS for veins) were accurately predicted in more than $90 \%$ of cases. Prediction accuracy holds true when considering not only the main conflicting vessel (i.e., the one with the highest grade of conflict found at surgery) but also all conflicting vessels (i.e., with the additional conflicts in the patients with multiple NVCs). With regard to arterial conflicts, the presence of SCA NVCs was rarely missed (sensitivity 92\%) but not infrequently overestimated (specificity 62\%). This is not surprising, since elongated SCAs are classically the mostly frequently encountered source of NVC and exert compression at the TREZ and/or in cisternal portions. ${ }^{32}$ Conversely, a frequent error was an underestimation of the involvement of the AICA (sensitivity 33\%), despite a high specificity (98\%). The value of Cohen's kappa for the AICA was 0.4; this low value may be due to the fact that the site of AICA-related compression was frequently at the TREZ/brainstem, where it was less obvious to the examiner. This is a factor to be remembered when examining patients' MR images.

Prediction of the venous NVCs was not so inaccurate when compared with prediction of the (overall) arterial NVCs, with no major over- or underestimation. This is probably due to the inclusion in our protocol of the highresolution gadolinium-enhanced T1-weighted sequence, which is able to depict veins in addition to arteries. Importantly, NVCs of venous origin were situated not only 
at the TREZ and/or cisternal portions but also at the porus trigeminus. In the latter situation, the vein was a deep superior petrosal vein of the transverse type, as pointed out previously elsewhere ${ }^{16}$ (Table 3 ).

The location of conflict was poorly predicted, with a low overall Cohen's kappa (0.37). This is probably mostly because of poor anatomical differentiation between conflicts situated at the TREZ and those situated in the cisternal portion of the nerve. While a clear microscopic definition of the TREZ has been made, ${ }^{1,12}$ MRI criteria to delimit the TREZ from the cisternal portion do not exist. This probably accounts for most of the inaccuracy in defining this difference. On the contrary, conflicts situated at the porus of Meckel's cave were accurately predicted. This was because this location was systematically looked at for potential conflicts on the T1 gadolinium MRI sequence. ${ }^{16}$

Grading the conflict proved to be a difficult task, with a low Cohen's kappa $(0.356,95 \%$ CI 0.350-0.362). Globally, the conflicts seen on MRI had an average grade significantly lower than that found at surgery, and only the grades of a minority of conflicts were precisely estimated. Nonetheless, prediction of the existence of a conflict with grade I seen on MRI as the cutoff was nearly perfect (area under the ROC curve 0.93). Additionally, the positive predictive value of seeing a grade II or III conflict on the MRI also approached $100 \%$. In fact, the most significant errors were undergrading conflicts of grade II or III. This would imply that some patients might be deprived of surgery while harboring a significant compression. Overgrading of MRI NVRs representing mere closeness or simple contact at surgery was a rarer occurrence than undergrading of high-grade conflicts. This makes us believe that a grade I conflict is, with current state-of-the-art MRI, the best compromise for a cutoff to define the conflict on imagery.

In this study, NVRs were looked for on both sides without knowing the side of the neuralgia; NVRs were identified on both sides in $44 \%$ of patients. This is surprising, but it confirms data from previous series. ${ }^{2,7,30}$ In some patients, it is clear that on the contralateral side of the neuralgia the finding was only a simple NVR with little or no compressive effect; this was found in the asymmetrical group, where the average grade was significantly lower than that on the ipsilateral side. But this was not the case in other patients.

In the symmetrical group, it was hard to determine the side of the patient's neuralgia because of almost identical images on both sides. In these patients, statistical analysis did not demonstrate a difference between the 2 sides when analyzing Cohen's kappa for the vessel involved, the site of compression, or the grade. However, many low-grade conflicts may have been overgraded, leading us to believe that some NVRs on the side contralateral to the neuralgia were considered conflicts while not actually compressive.

In contrast, errors in predicting the side of the neuralgia were highly infrequent when an NVR was seen on only 1 side $(5 \%)$. These errors were made only in those patients in whom no actual conflict was found at surgery on the side of the neuralgia. This $5 \%$ error rate was significantly lower than the rate in the patients whose MR images were interpreted as showing bilateral NVRs (36\%), and the difference was even greater when the comparison was to those whose MR images were interpreted as showing symmetrical NVRs (43\%).

Previous studies addressing the sensitivity and specificity of MRI have shown excellent concordance between surgical findings and MRI examination. ${ }^{3-5,30}$ However, in many of those studies the examiner(s) was (were) not completely blinded to all clinical data and/or surgical findings, including the side of the neuralgia. ${ }^{9,34}$ It is self-evident that not allowing access to any information about the clinical situation reduces the accuracy of the evaluation. Nonetheless, under these conditions in our study, $50 \%$ of the cases that had been operated on with a postulated true conflict were identified as negative (true negatives).

In many published MRI studies, NVRs have been described in patients who do not have any symptoms of TN or on the side contralateral to the side of the pain. ${ }^{26,31,35}$ Our study provides additional support for these findings. However, the incertitude pointed out in determining the true nature of an NVR seen on the MRI study to be of grade I suggests that many of these images outside of the clinical setting are just that: NVRs, not necessarily conflicts, but merely closeness between the vessel and the nerve.

Overall, this study shows a good capability of MRI to predict the presence of a conflict, accurately predicting the vessel involved, but with a significant uncertainty in determining the grade of the conflict shown to have an important impact in the process of surgical decision making. ${ }^{38}$

The types of MRI and sequences used to obtain diagnostic reliability have been analyzed in several publications..$^{8,27}$ Some authors rely on 3D high-resolution T2-weighted images, others advocate combining T2-weighted sequences with MR angiography, ${ }^{5}$ and yet others prefer T2-weighted and T1-weighted sequences with gadolinium. On our part, we stress the importance of the 3 following sequences: high-resolution 3D T2-weighted sequences, TOF angiography, and 3D T1-weighted sequences with gadolinium to increase the sensitivity and improve the specificity. ${ }^{23}$ This combination allows the detection of purely venous compressions or veins associated with arterial conflicts, as well as the differentiation of their exact anatomical origin. Pure venous compression or compressive veins associated with arterial NVC were found in $13 \%$ and $26 \%$ of our cases, respectively, similar to rates previously reported in the literature. ${ }^{16}$

Although some publications have investigated the capability of MRI to identify the nature of the conflicting vessel, very few have studied the reliability of MRI in predicting the degree of compression. ${ }^{8,26}$

The most important information revealed by this study is probably not the morphological description of the conflicts but the decision-making details when facing a patient to whom a surgical intervention may be proposed. It turns out that utilizing a grade I cutoff probably offers the best balance between sensitivity and specificity. However, this does imply that a certain number of patients will undergo surgical exploration only to learn that no real conflict was present. In the present series, this occurred only in patients with low-grade conflicts seen on MRI, whereas when a high-grade conflict was seen on imaging, a true conflict was almost certain to have been found at surgery.

In view of the differential results of decompression according to grade (i.e., the good results at high grades of 
conflict-between $75 \%$ and $85 \%$ at 15 years), ${ }^{38}$ one should probably have little hesitation when suggesting surgery to a patient whose imaging studies appear to show a highgrade conflict.

On the other hand, when the MRI interpretation is that a low-grade conflict (grade I) is present, one can expect any type of NVR, as shown by the results of this study. The absence of compression is rare, but it has been encountered in this series, while a significant number of patients who were thought to have a grade I conflict based on MRI actually were found to have important compressions (grade II or III) at surgery. This is what makes surgical indication when facing an MR image showing a grade I conflict such a difficult choice. Especially when facing an MR image showing grade I, other factors-such as patients' general status, their clinical presentation, and the divisions involved-should weigh heavily in the balance, together with a critical discussion of what other methods may be appropriate in that situation.

Other methods for the diagnosis of a true conflict-although not in common clinical practice-are especially useful when dealing with grade I conflicts. Using measurements of the volume of the nerve to determine whether a focal atrophy is present is one such possibility. Focal atrophy has been proved to be associated with real conflicts and good surgical outcomes. ${ }^{13,17,22,33}$ In cases of simple contact, however, there may be no focal atrophy (otherwise, the conflict should be graded as grade II or III), and this method may therefore not be easily usable in this context. Multidimensional reconstruction of the nerve might be helpful, especially in identifying indentations or deformations of the nerve and in that sense differentiating between grade I and grade II. ${ }^{36}$ However, since the key problem is to identify which cases of apparent grade I conflict are true conflicts and which are not, both methods may be unsatisfactory in this respect. Alternatively, the use of diffusion tensor imaging with fractional anisotropy and diffusivity values may help in determining whether a true conflict with nerve parenchyma alteration is present. ${ }^{14,15,22,24}$ This method may be less dependent on the degree of conflict on imaging, and alteration of the fibers inside the nerve should in theory make a connection between the vascular contact and the neuralgia. The drawbacks of this method are the amount of time it consumes and its resource cost.

\section{Conclusions}

On a practical level, our study shows that the finding of likely NVC on MRI is a solid argument for considering MVD. This applies particularly to cases of high-grade compression, for which there is a high probability that patients will experience long-term relief of TN symptoms after the procedure. When dealing with patients whose MRI shows simple contact, the decision between MVD and alternative lesioning methods should be made on a case-by-case basis after careful consideration of factors related to the patient's general condition, as well as their TN symptoms.

\section{References}

1. Adams CB: Microvascular compression: an alternative view and hypothesis. J Neurosurg 70:1-12, 1989
2. Alper J, Shrivastava RK, Balchandani P: Is there a magnetic resonance imaging-discernible cause for trigeminal neuralgia? A structured review. World Neurosurg 98:89-97, 2017

3. Anderson VC, Berryhill PC, Sandquist MA, Ciaverella DP, Nesbit GM, Burchiel KJ: High-resolution three-dimensional magnetic resonance angiography and three-dimensional spoiled gradient-recalled imaging in the evaluation of neurovascular compression in patients with trigeminal neuralgia: a double-blind pilot study. Neurosurgery 58:666-673, 2006

4. Antonini G, Di Pasquale A, Cruccu G, Truini A, Morino S, Saltelli G, et al: Magnetic resonance imaging contribution for diagnosing symptomatic neurovascular contact in classical trigeminal neuralgia: a blinded case-control study and metaanalysis. Pain 155:1464-1471, 2014

5. Boecher-Schwarz HG, Bruehl K, Kessel G, Guenthner M, Perneczky A, Stoeter P: Sensitivity and specificity of MRA in the diagnosis of neurovascular compression in patients with trigeminal neuralgia. A correlation of MRA and surgical findings. Neuroradiology 40:88-95, 1998

6. Borgaro SR, Gierok S, Caples H, Kwasnica C: Fatigue after brain injury: initial reliability study of the BNI Fatigue Scale. Brain Inj 18:685-690, 2004

7. Burchiel KJ: Trigeminal neuralgia: new evidence for origins and surgical treatment. Neurosurgery 63 (Suppl 1):52-55, 2016

8. Chen MJ, Zhang WJ, Guo ZL, Yang C, Zhang WH, Dong MJ, et al: Preoperative evaluation of the neurovascular compression using magnetic resonance tomographic angiography: our radiologic indications for microvascular decompression to treat trigeminal neuralgia. J Craniofac Surg 25:e384e388, 2014

9. Chun-Cheng Q, Qing-Shi Z, Ji-Qing Z, Zhi-Gang W: A single-blinded pilot study assessing neurovascular contact by using high-resolution MR imaging in patients with trigeminal neuralgia. Eur J Radiol 69:459-463, 2009

10. Cohen J: A coefficient of agreement for nominal scales. Educ Psychol Meas 20:37-46, 1960

11. Cruccu G, Finnerup NB, Jensen TS, Scholz J, Sindou M, Svensson P, et al: Trigeminal neuralgia: new classification and diagnostic grading for practice and research. Neurology 87:220-228, 2016

12. De Ridder D, Møller A, Verlooy J, Cornelissen M, De Ridder L: Is the root entry/exit zone important in microvascular compression syndromes? Neurosurgery 51:427-434, 2002

13. DeSouza DD, Hodaie M, Davis KD: Abnormal trigeminal nerve microstructure and brain white matter in idiopathic trigeminal neuralgia. Pain 155:37-44, 2014

14. DeSouza DD, Hodaie M, Davis KD: Structural magnetic resonance imaging can identify trigeminal system abnormalities in classical trigeminal neuralgia. Front Neuroanat 10:95, 2016

15. Duan Y, Sweet J, Munyon C, Miller J: Degree of distal trigeminal nerve atrophy predicts outcome after microvascular decompression for Type 1a trigeminal neuralgia. J Neurosurg 123:1512-1518, 2015

16. Dumot C, Brinzeu A, Berthiller J, Sindou M: Trigeminal neuralgia due to venous neurovascular conflicts: outcome after microvascular decompression in a series of 55 consecutive patients. Acta Neurochir (Wien) 159:237-249, 2017

17. Erbay SH, Bhadelia RA, O'Callaghan M, Gupta P, Riesenburger R, Krackov W, et al: Nerve atrophy in severe trigeminal neuralgia: noninvasive confirmation at MR imaging-initial experience. Radiology 238:689-692, 2006

18. Hallgren KA: Computing inter-rater reliability for observational data: an overview and tutorial. Tutor Quant Methods Psychol 8:23-34, 2012

19. Hanley JA, McNeil BJ: The meaning and use of the area under a receiver operating characteristic (ROC) curve. Radiology 143:29-36, 1982

20. Lang E, Naraghi R, Tanrikulu L, Hastreiter P, Fahlbusch R, 
Neundörfer B, et al: Neurovascular relationship at the trigeminal root entry zone in persistent idiopathic facial pain: findings from MRI 3D visualisation. J Neurol Neurosurg Psychiatry 76:1506-1509, 2005

21. Lasko TA, Bhagwat JG, Zou KH, Ohno-Machado L: The use of receiver operating characteristic curves in biomedical informatics. J Biomed Inform 38:404-415, 2005

22. Leal PRL, Barbier C, Hermier M, Souza MA, Cristino-Filho $\mathrm{G}$, Sindou M: Atrophic changes in the trigeminal nerves of patients with trigeminal neuralgia due to neurovascular compression and their association with the severity of compression and clinical outcomes. J Neurosurg 120:1484-1495, 2014

23. Leal PRL, Hermier M, Froment JC, Souza MA, CristinoFilho G, Sindou M: Preoperative demonstration of the neurovascular compression characteristics with special emphasis on the degree of compression, using high-resolution magnetic resonance imaging: a prospective study, with comparison to surgical findings, in 100 consecutive patients who underwent microvascular decompression for trigeminal neuralgia. Acta Neurochir (Wien) 152:817-825, 2010

24. Leal PRL, Roch JA, Hermier M, Souza MAN, Cristino-Filho G, Sindou M: Structural abnormalities of the trigeminal root revealed by diffusion tensor imaging in patients with trigeminal neuralgia caused by neurovascular compression: a prospective, double-blind, controlled study. Pain 152:23572364, 2011

25. Maarbjerg S, Sørensen MT, Gozalov A, Bendtsen L, Olesen J: Field-testing of the ICHD-3 beta diagnostic criteria for classical trigeminal neuralgia. Cephalalgia 35:291-300, 2015

26. Maarbjerg S, Wolfram F, Gozalov A, Olesen J, Bendtsen L: Significance of neurovascular contact in classical trigeminal neuralgia. Brain 138:311-319, 2015

27. Masuda Y, Yamamoto T, Akutsu H, Shiigai M, Masumoto T, Ishikawa E, et al: Usefulness of subtraction of 3D T2WIDRIVE from contrast-enhanced 3D T1WI: preoperative evaluations of the neurovascular anatomy of patients with neurovascular compression syndrome. AJNR Am J Neuroradiol 36:317-322, 2015

28. McCartney S, Weltin M, Burchiel KJ: Use of an artificial neural network for diagnosis of facial pain syndromes: an update. Stereotact Funct Neurosurg 92:44-52, 2014

29. Meaney JF, Eldridge PR, Dunn LT, Nixon TE, Whitehouse $\mathrm{GH}$, Miles JB: Demonstration of neurovascular compression in trigeminal neuralgia with magnetic resonance imaging. Comparison with surgical findings in 52 consecutive operative cases. J Neurosurg 83:799-805, 1995

30. Miller J, Acar F, Hamilton B, Burchiel K: Preoperative visualization of neurovascular anatomy in trigeminal neuralgia. $\mathbf{J}$ Neurosurg 108:477-482, 2008

31. Miller JP, Acar F, Hamilton BE, Burchiel KJ: Radiographic evaluation of trigeminal neurovascular compression in patients with and without trigeminal neuralgia. J Neurosurg 110:627-632, 2009

32. Mistry AM, Niesner KJ, Lake WB, Forbes JA, Shannon $\mathrm{CN}$, Kasl RA, et al: Neurovascular compression at the root entry zone correlates with trigeminal neuralgia and early microvascular decompression outcome. World Neurosurg 95:208-213, 2016

33. Park SH, Hwang SK, Lee SH, Park J, Hwang JH, Hamm IS:
Nerve atrophy and a small cerebellopontine angle cistern in patients with trigeminal neuralgia. J Neurosurg 110:633637, 2009

34. Patel NK, Aquilina K, Clarke Y, Renowden SA, Coakham HB: How accurate is magnetic resonance angiography in predicting neurovascular compression in patients with trigeminal neuralgia? A prospective, single-blinded comparative study. Br J Neurosurg 17:60-64, 2003

35. Peker S, Dinçer A, Necmettin Pamir M: Vascular compression of the trigeminal nerve is a frequent finding in asymptomatic individuals: 3-T MR imaging of 200 trigeminal nerves using 3D CISS sequences. Acta Neurochir (Wien) 151:1081-1088, 2009

36. Satoh T, Omi M, Nabeshima M, Onoda K, Date I: Severity analysis of neurovascular contact in patients with trigeminal neuralgia: assessment with the inner view of the 3D MR cisternogram and angiogram fusion imaging. AJNR Am J Neuroradiol 30:603-607, 2009

37. Sindou M, Howeidy T, Acevedo G: Anatomical observations during microvascular decompression for idiopathic trigeminal neuralgia (with correlations between topography of pain and site of the neurovascular conflict). Prospective study in a series of 579 patients. Acta Neurochir (Wien) 144:1-13, 2002

38. Sindou M, Leston J, Decullier E, Chapuis F: Microvascular decompression for primary trigeminal neuralgia: long-term effectiveness and prognostic factors in a series of 362 consecutive patients with clear-cut neurovascular conflicts who underwent pure decompression. J Neurosurg 107:1144-1153, 2007

39. Wäljas M, Iverson GL, Hartikainen KM, Liimatainen S, Dastidar P, Soimakallio S, et al: Reliability, validity and clinical usefulness of the BNI fatigue scale in mild traumatic brain injury. Brain Inj 26:972-978, 2012

40. Zou KH, O’Malley AJ, Mauri L: Receiver-operating characteristic analysis for evaluating diagnostic tests and predictive models. Circulation 115:654-657, 2007

41. Zweig MH, Campbell G: Receiver-operating characteristic (ROC) plots: a fundamental evaluation tool in clinical medicine. Clin Chem 39:561-577, 1993

\section{Disclosures}

The authors report no conflict of interest concerning the materials or methods used in this study or the findings specified in this paper.

\section{Author Contributions}

Conception and design: Brînzeu. Acquisition of data: all authors. Analysis and interpretation of data: all authors. Drafting the article: Brînzeu. Critically revising the article: Brînzeu, Sindou. Reviewed submitted version of manuscript: Brînzeu, Sindou. Approved the final version of the manuscript on behalf of all authors: Brînzeu. Statistical analysis: Brînzeu, Drogba. Administrative/technical/material support: Brînzeu. Study supervision: Brînzeu.

\section{Correspondence}

Andrei Brînzeu: Hôpital Neurologique, Lyon, France. andrei. brinzeu@chu-lyon.fr. 